\title{
Coronary artery fistulas: A case presentation and literature review
}

\author{
Mark Chou $^{1}$, Helme Silvet ${ }^{1,2}$, Gaurav Tyagi $^{1,2}$ \\ 1. Department of Internal Medicine, Loma Linda University, California, United States. 2. Department of Cardiology, Loma \\ Linda Veterans Affairs Hospital, California, United States.
}

Correspondence: Mark Chou. Address: 74 Sunflower St, Redlands CA 92373, United States. Email: mchou@llu.edu

Received: July 24, 2014

DOI : $10.5430 /$ crim.v2n1p1

\begin{abstract}
Coronary fistulas are rare anomalies that connect the coronary artery to either a cardiac chamber (coronary cameral fistula) or vein (arteriovenous fistula). They arise due to abnormalities in embryologic development and are often coincidental findings. The exact incidence is estimated to be between 0.1-0.2 percent of the general population. There are no consensus guidelines for management of these fistulas. We present a patient with a large fistula and will review the literature and management decisions for this clinical abnormality.
\end{abstract}

A 66-year-old man with diabetes, hypertension and a prior bioprosthetic aortic valve presented to the Loma Linda VA Hospital with shortness of breath. An echocardiogram revealed moderate to severe prosthetic aortic stenosis, and an angiogram showed $80 \%$ mid left anterior descending (LAD) obstruction with a large arteriovenous fistula. The fistula extended from the circumflex artery to coronary sinus. He was referred for redo open heart surgery with aortic valve replacement, coronary bypass grafting and repair of the fistula.

There are multiple case reports of these malformations causing symptoms of angina, coronary steal or heart failure based on various testing modalities. However, in 31 individuals with incidental findings of coronary fistulas from a small case series, no adverse effects on cardiac function were noted based on physical exam or functional testing. If treatment is deemed beneficial, surgery is the most available option and another study of 41 symptomatic patients reported low post-operative morbidity and mortality after nine-year follow-up.

The authors believe that large fistulas found incidentally should be further evaluated for ischemia and monitored for symptoms. For individuals with small fistulas, conservative management is a viable strategy as there is a high incidence of spontaneous closure. If a fistula is determined to be symptomatic, operative repair is well tolerated.

\section{Keywords}

Coronary artery fistula, Coronary cameral fistula, Arteriovenous fistula

\section{I ntroduction}

Coronary fistulae are rare anomalies that connect the coronary artery to either a cardiac chamber, known as a coronary cameral fistula (CCF), or any of the great vessels (coronary sinus, pulmonary artery, superior vena cava or pulmonary 
veins), called coronary arteriovenous fistula. Coronary artery fistulae (CAF) were first described by Krause in 1865 . Abott described them in more detail in 1906, and the first successful surgical closure was described by Bjork and Crafoord in $1947^{[1]}$.

Coronary artery fistulae arise due to abnormalities in embryonic development of the coronary circulation. Normal arteries originate as an endothelial outgrowth in the base of the aorta and communicate with the capillary network of the surface of the heart. The intramyocardial sinusoids become narrowed and persist only as thebesian vessels in adults. If the intramyocardial trabecular sinusoids fail to obliterate, a fistulous communication persists between the arteries and a cardiac chamber or vein ${ }^{[2]}$. Acquired coronary artery fistulas can occur from trauma, infection, surgical repairs for congenital abnormalities, endomyocardial biopsies, and percutaneous coronary interventions ${ }^{[1]}$.

Coronary fistulas are often coincidental findings on cardiac evaluation. The exact incidence is unknown but estimated to be between 0.1-0.2 percent of the general population ${ }^{[3]}$. Based on a case series by Huang, most coronary artery fistulas originated from the right coronary artery ( $50 \%$ to $58 \%$ of cases); followed by left anterior descending artery (25\%), circumflex artery (18\%), diagonal (2\%), and rarely from left main (<1\%). They drained into the pulmonary artery in $30 \%$ to $43 \%$ of cases, right ventricle in $14 \%$ to $40 \%$, right atrium in $19 \%$ to $20 \%$, left ventricle in $6 \%$ to $19 \%$, and left atrium in $5 \%$ of cases ${ }^{[4]}$.

Most patients with coronary fistulas are asymptomatic but coronary steal or shunting physiology can result in symptoms due to acute coronary syndromes or heart failure ${ }^{[5]}$. Infective endocarditis is a less frequent complication ${ }^{[6]}$. There are multiple case reports confirming these symptoms on stress testing and even event monitoring ${ }^{[7]}$.

We present a case of a patient with a large AV fistula and will review the literature and management decisions for this clinical abnormality.

\section{Case presentation}

Our patient was a 66 year old non smoking man with diabetes, hypertension and a prior bioprosthetic aortic valve replacement in 2001 due to endocarditis. He presented to the Loma Linda Veterans Hospital with progressive dyspnea on exertion. He was admitted to the cardiac care unit for evaluation and received an echocardiogram that revealed moderate to severe prosthetic aortic stenosis (see Figure 1). Preoperative cardiac catheterization was performed which showed $80 \%$ mid LAD obstruction and a large AV fistula branching from the left main coronary artery (see Figure 2). Contrast was diluted in the fistula making the images suboptimal. Cardiac computer tomography (CT) was performed to better characterize the anomaly and estimated the diameter of the fistula to be $9.6 \mathrm{~mm}$ with a tortuous path from the proximal circumflex artery to the coronary sinus (see Figure 3, 4, 5). He was referred for redo open heart surgery with aortic valve replacement, coronary bypass surgery and repair of the fistula. Intraoperatively, single vessel bypass grafting was performed to the LAD followed by replacement of the deteriorated mechanical valve. The coronary sinus was unroofed through the right atrium and the fistula termination point was ligated. He was also found to have a patent foramen ovale that was undetected on imaging and this was closed as well. After the operation, he was followed as an outpatient without further symptoms.

Due to multiple reasons for the patient's symptoms, it was never clearly elucidated whether any symptoms were due to the large fistula. The decision to ligate the fistula was made solely based on the size of the fistula and other pathology necessitating open heart surgery.

He was re-evaluated five years later due to a transient episode of shortness of breath but no significant change in cardiac chamber size or in cardiac function was found on nuclear stress testing or echocardiography. Repeat angiography was 
performed and the proximal circumflex artery remained ectactic but the fistula was no longer seen. The surgery was therefore considered successful.

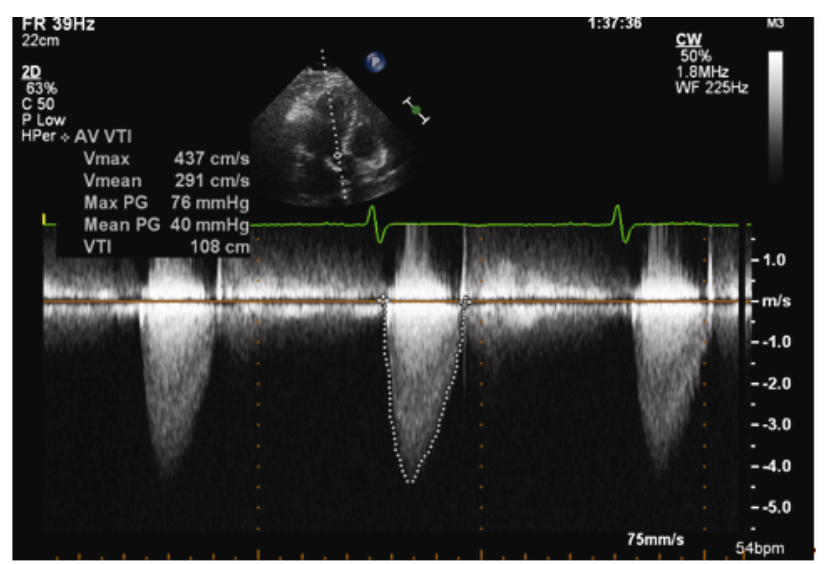

Figure 1. 2D Echo, Continuous Wave Doppler - Severe prosthetic aortic stenosis, mean gradient $40 \mathrm{mmHg}$

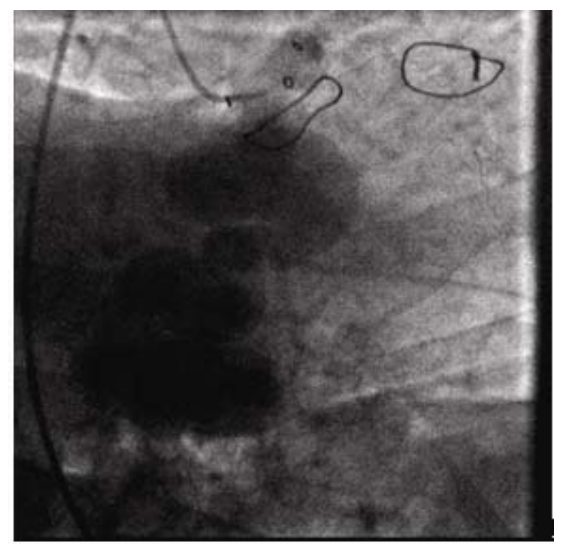

Figure 2. Coronary Angiogram, 23 CAU 37 RAO - Large fistula visualized when engaging left sided circulation

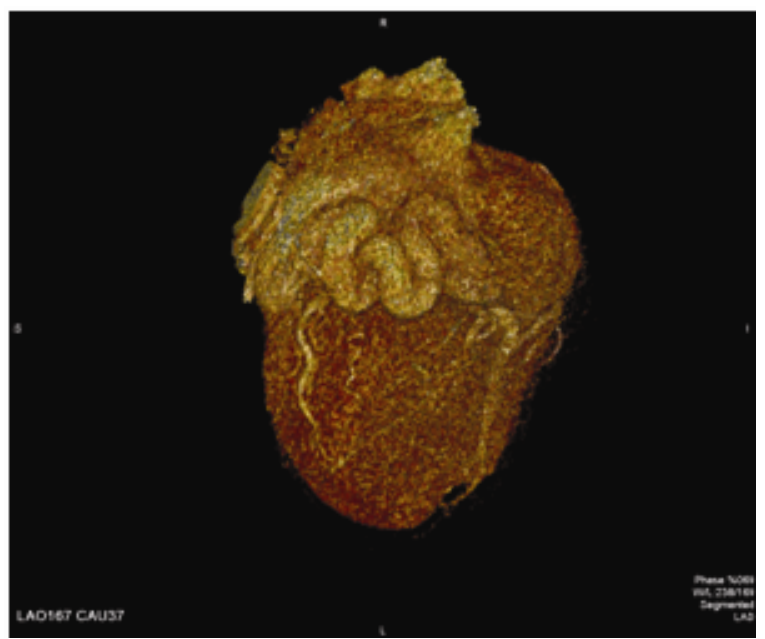

Figure 3. Cardiac CT 3D Volume Rendered Image-Large coronary fistula coursing in the posterior atrioventricular groove
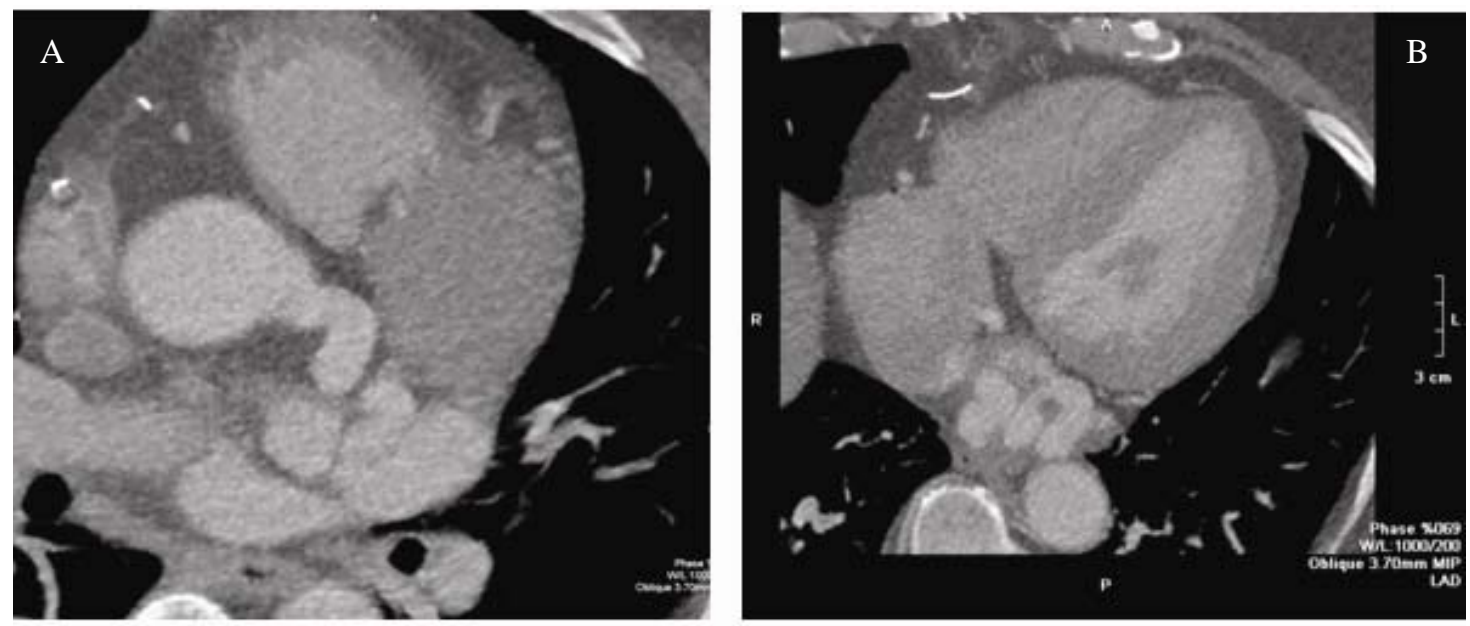

Figure 4. Multiplanar Reconstruction, transverse view (A) dilated proximal left circumflex gives rise to the AV fistula which courses in the posterior atrioventricular groove (B) 

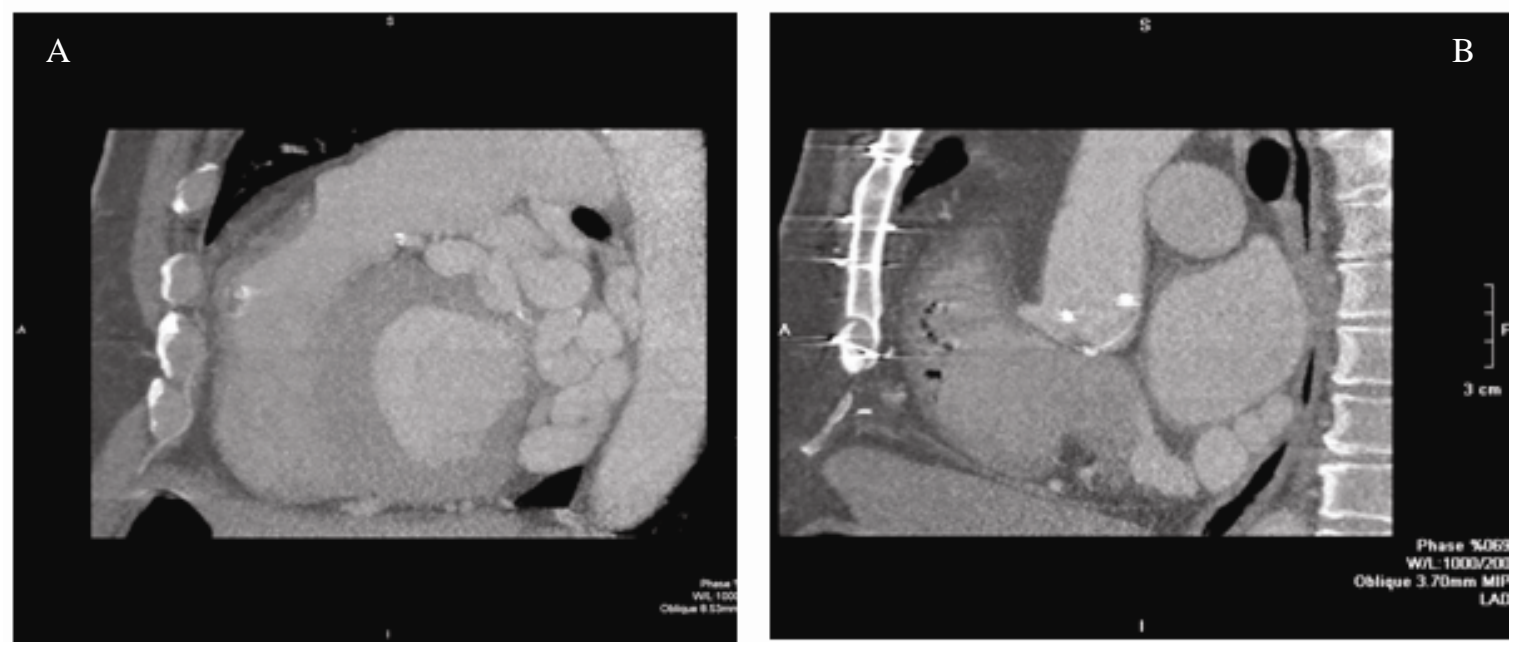

Figure 5. Multiplanar Reconstruction, sagittal view. AV fistula again coursing in the posterior atrioventricular groove (A) before entering the dilated coronary sinus (B)

\section{Discussion}

Coronary artery fistulae are rare entities that do not have strict management algorithms. Prognosis, diagnosis and treatment options vary on a case by case basis.

Hemodynamic derangements related to coronary fistulas depend on size, tortuosity and the chamber of communication. Fistulas emptying into right atrium or right ventricle cause a volume overload on the right heart, whereas fistulas terminating in the pulmonary artery overload the pulmonary circulation and left ventricle as a result of the left-to-right shunt ${ }^{[2]}$.

The natural history of coronary fistulae is often benign as they are usually found coincidentally and are commonly asymptomatic. Sherwood et al monitored 31 individuals with incidental finding of coronary fistulae and no adverse effects on cardiac function were noted based on physical exam or functional testing. Spontaneous closures of the malformations were noted in nearly $25 \%$ of cases based on repeat echocardiography with Doppler ${ }^{[8]}$. Raju et al stated that various patient factors contribute to development of symptoms including age, amount of shunting and development of ischemia. The most common symptom from these fistulae is chest pain attributed to coronary steal ${ }^{[9]}$. Coronary artery fistulae can become quite impressive with the largest one documented by Gupta et al ${ }^{[3]}$. This fistula was found to be $6.0 \mathrm{~cm} \times 5.6 \mathrm{~cm}$ $\times 4.8 \mathrm{~cm}$ on cardiac CT in an elderly female complaining of dyspnea. The progression of fistulae due to inflammatory states is not documented.

Auscultation usually reveals a continuous murmur, different from the location of that of patent ductus arteriosus. Electrocardiogram is usually normal but may show right or left ventricular overload. In many cases two dimensional echocardiography with Doppler analysis can identify the origin and termination site, as well as chamber enlargement and overload. In some cases a transesophageal echocardiography may be needed to ascertain the precise measurements and location of fistula to help plan the surgical intervention. Multi-detector Cardiac CT has become the non-invasive test of choice for detection of cardiac anomalies. CT helps delineate the entire course of the fistula in relation to adjacent cardiac chambers.

However, cardiac catheterization and selective coronary angiography is usually needed to make a definitive diagnosis ${ }^{[9]}$. Precise determination of shunt flow (Qp/Qs), degree of cardiac decompensation and assessment of concomitant coronary artery disease can be performed simultaneously. If shunt flow is significant, it may lead to increased left ventricular 
end-diastolic pressure, left ventricular hypertrophy and congestive heart failure. Valvular regurgitation due to papillary muscle dysfunction and infective endocarditis of regurgitant valves has been described ${ }^{[1]}$.

Provocative testing can also be performed, if clinical suspicion warrants, and a case series of 5 individuals by Said et al described various modalities to look at myocardial perfusion from SPECT imaging to cardiac magnetic resonance imaging and even positron emission tomography (PET) to detect coronary steal ${ }^{[10]}$. Koh et al documented a symptomatic fistula by noting ST segment changes on an event monitor in a patient with a known fistula ${ }^{[7]}$.

Most asymptomatic patients can be observed clinically. If a coronary fistula is symptomatic and treatment is deemed beneficial, surgery is the most available option. Cheung et al followed 41 symptomatic patients after surgery and reported no post-operative mortality after nine-year average follow-up ${ }^{[11]}$. There was overall low morbidity and no episodes of myocardial infarction. However, four of the individuals had persistent fistulas at follow up.

Another treatment modality is percutaneous closure and a small cohort by McMahon et al of four pediatric patients underwent time released coil closure ${ }^{[12]}$. Complete fistula occlusion occurred in two of four patients at three years of follow up. One individual required surgical occlusion a year later and the final patient had residual fistula but no intervention was necessary. Various types of occlusion coils, vascular plugs, covered stents, umbrella devices, detachable balloons and histoacryl resin have been used ${ }^{[1]}$.

In summary, coronary artery fistulas are usually benign conditions that is found incidentally on cardiac evaluation. Diagnosis can be made with coronary angiography, cardiac CT or echocardiography with Doppler. The fistulas can become quite large and it is reasonable to further evaluate individuals by stress testing modalities to provoke symptoms, or by event monitoring. For small fistulas, conservative management is a viable strategy as there is a high incidence of spontaneous closure. If treatment is determined to be beneficial, operative repair appears to be well tolerated with low morbidity and mortality. Although percutaneous closure is possible, provider experience is limited and complications may arise. In our individual and others with a separate indication for cardiothoracic surgery, repair can often be done without the need to isolate further symptoms.

\section{References}

[1] Mangukia CV. Coronary artery fistula. Ann Thorac Surg. 2012 Jun; 93(6): 2084-92. http://dx.doi.org/10.1016/j.athoracsur.2012.01.114

[2] Rittenhouse EA, Doty DB, Ehrenhaft JL. Congenital coronary artery-cardiac chamber fistula: review of operative management. Ann Thorac Surg. 1975; 20: 468-485. http://dx.doi.org/10.1016/S0003-4975(10)64245-2

[3] Gupta Vishal, Truong Quynh, Okada David. et al. Giant Left Circumflex Coronary Artery Aneurysm with Arteriovenous Fistula to the Coronary Sinus. Circulation 2008; 118: 2304. http://dx.doi.org/10.1161/CIRCULATIONAHA.108.781617

[4] Huang YK, Lei MH, Lu MS, et al. Bilateral coronary-to-pulmonary artery fistulas. Ann Thorac Surg. 2006; 82: 1886-1888. http://dx.doi.org/10.1016/j.athoracsur.2006.02.040

[5] Kasper, et al. Congenital Heart Disease in the Adult. Harrisons Principals of Internal Medicine 17th edition 2008. 1461.

[6] Kasravi Babak, Reid Cheryl L, Allen Byron. Coronary Artery Fistula Presenting as Bacterial Endocarditis. J Am Soc Echocardiogr. 2004; 17: 1315 - 16. http://dx.doi.org/10.1016/j.echo.2004.06.028

[7] Koh Kwang Kon, Cho Sang Kyoon, Kim Sam Soo. Left and Right Coronary Artery to Left Ventricular Fistula: Demonstration of Myocardial ischemic on treadmill test and holter monitoring - a case report. Angiology. 1993; 44: 977.

http://dx.doi.org/10.1177/000331979304401209

[8] Sherwood Megan C, Rockenmacher Sol, Colan Steven D, Geva Tal. Prognostic Significance of Clinically Silent Coronary Artery Fistulas. Am J of Cardiol. 1999; 83: 407-411. http://dx.doi.org/10.1016/S0002-9149(98)00878-9

[9] Raju Manjunath G, Goyal Sandeep K, Punnam Sujeeth R, et al. Coronary Artery Fistula: A Case Series with Review of the Literature. J of Cardiol. 2009; 53: 467-472. http://dx.doi.org/10.1016/j.jjcc.2008.09.009 
[10] Said SAM, Nijhuis RLG, op den Akker JW, et al. Diagnostic and therapeutic approach of Congenital Solitary Coronary Artery Fistulas in Adults: Dutch Case Series and Review of Literature. Neth Heart J. 2011; 19: 183-191. http://dx.doi.org/10.1007/s12471-011-0088-2

[11] Cheung LC, Au WK, Cheung HHC, et al. Coronary Artery Fistulas: Long-Term Results of Surgical Correction. Ann Thorac Surg. 2001; 71: 190-5. http://dx.doi.org/10.1016/S0003-4975(00)01862-2

[12] McMahon, Colin J, Nihill Michael, et al. Coronary Artery Fistula: Management and Intermediate-Term Outcome after Transcatheter Closure. Tex Heart Inst J. 2001; 28(1): 21-25. 